\title{
Resampling Reveals Sample-Level Differential Expression in Clinical Genome-Wide Studies
}

\author{
Jukka Hiissa, ${ }^{1,2}$ Laura L. Elo,,2 Kaisa Huhtinen,,4 Antti Perheentupa, ${ }^{3,4}$ \\ Matti Poutanen, ${ }^{4,5}$ and Tero Aittokallio ${ }^{1,2}$
}

\begin{abstract}
Genome-scale molecular profiling of clinical sample material often results in heterogeneous datasets beyond the capability of standard statistical procedures. Statistical tests for differential expression, in particular, rely upon the assumption that the sample groups being compared are relatively homogeneous. Such assumption rarely holds in clinical materials, which leads to detection of secondary findings (false positives) or loss of significant targets (false negatives). Here, we introduce a resampling-based procedure, named ReScore, which aggregates individual changes across all the samples while preserving their clinical classes, and thereby provides multiple sets of markers that can effectively characterize distinct sample subsets. When applied to a public leukemia microarray study, the procedure could accurately reveal hidden subgroup structures associated with underlying genotypic abnormalities. The procedure improved both the sensitivity and specificity of the findings, as well as helped us to identify several disease subtype-specific genes that have remained undetected in the conventional analyses. In our endometriosis study, we were able to accurately distinguish between various sources of systematic variation, linked, for example, to tissue-specificity and disease-related factors, many of which would have been missed with standard approaches. The generic procedure should benefit also other global profiling experiments such as those based on mass spectrometry-based proteomic assays.
\end{abstract}

\section{Introduction}

G ENOME-WIDE PROFILING TECHNOLOGIES, such as gene expression microarrays and mass spectrometry-based proteomic assays, are increasingly being applied to address clinical research questions. Characteristic expression patterns revealed across different clinical conditions and/or time points offer the possibility, for example, to compose a set of diagnostic markers for disease screening or early prognosis, reveal potential targets for drug development, and eventually illuminate the molecular mechanisms underlying the disease progression. The clinical feasibility of global microarray screens has been demonstrated, for example, in the context of breast cancer prediction (van't Veer et al., 2002; Wittner et al., 2008). However, other clinical applications have remained relatively rare, despite various high-throughput profiling efforts in a wide range of diseases both at the mRNA and protein level (Schmidt and Aebersold, 2006; Wang and Cheng, 2005).
Although it has become a routine to perform and analyze microarray hybridizations on carefully controlled sample material, a number of challenging problems remain in the analysis of more complex samples on a global scale. Most of the clinical data are from cross-sectional designs, which reflect multiple sources of biological and/or experimental variation that may severely confound the actual phenotypic variation under investigation. Such confounding factors often manifest in hidden sample subgroups, which are not predefined, but show systematic expression changes and may associate with divergent clinical outcomes. Experimental problems that may further complicate the resulting data sets include, for example, missing data values and sample labeling errors. Moreover, due to the high cost of these experiments and the scarcity of clinical samples, many studies are performed on relatively small sample sizes, compared to the large number of genes or proteins probed in the complex sample mixtures.

Despite many improvements in the experimental design and preprocessing of microarray experiments, and useful

\footnotetext{
${ }^{1}$ Biomathematics Research Group, Department of Mathematics, University of Turku, Turku, Finland.

${ }^{2}$ Data Mining Research Group, Turku Centre for Biotechnology, Turku, Finland.

${ }^{3}$ Department of Obstetrics and Gynecology, University of Turku, Turku, Finland.

${ }^{4}$ Department of Physiology, Institute of Biomedicine, University of Turku, Turku, Finland.

${ }^{5}$ Turku Center for Disease Modeling, University of Turku, Turku, Finland.
} 
modifications to the statistical tests for detecting differential expression (Allison et al., 2006; Efron and Tibshirani, 2002; Elo et al., 2008; Holzman and Kolker, 2004; Rotter et al. 2008; Tusher et al., 2001), the complex and multimodal nature of clinical datasets still poses severe problems to those statistical procedures that rely on the assumption that the classes being compared are relatively homogeneous. In particular, when the disease-related alterations are only subtle, the undetected interindividual and technical variability within the clinical classes together with the limited sample sizes make it difficult to distinguish those elements directly linked to the disease from innocent bystander elements whose expression has been altered by secondary effects or technical artefacts (Miklos and Maleszka, 2004). Consequently, it has been recognized that the panels of biomarkers extracted from the genome-scale screens can show very poor reproducibility across different studies or even between different subsamples in the same study (Ein-Dor et al., 2005; Michiels et al., 2005).

Toward exploiting the full clinical potential of the genomescale studies, we introduce here an efficient data-mining procedure for revealing differential expression in heterogeneous datasets. The aim of the procedure is to help the researchers to identify genes that show statistically significant differences between such sample subgroups that are hidden inside the given clinical classes while being relevant for the question under study. This aim is achieved by means of a novel resampling-based framework, which goes beyond the single panel of markers obtained using standard statistical testing. The procedure also takes advantage of the given class information that is lost in the traditional sample clustering methods. Using three heterogeneous microarray datasets of clinical specimens as case studies, we show how the procedure can overcome the fundamental limitations of the conventional analysis procedures and provide several novel findings with potential diagnostic and pharmaceutical implications.

\section{Materials and Methods}

\section{The generic ReScore procedure}

The operation of our generic analysis framework is organized through nine subsequent steps (Fig. 1, the blue arrows). The framework is built on resampling-based comparisons across clinical samples, through which the measured intensity data matrix is transformed into a more robust score matrix that carries information not only on the given sample classes (e.g., patients vs. controls), but also on other clinically relevant sample subgroups (e.g., disease subtypes). Compared with the conventional analysis procedures (the two dotted arrows), the resulting score matrix can greatly improve the subsequent data analysis phases, such as grouping of the clinical samples according to their individual score vectors when revealing clinically meaningful hidden subgroup structures with coherent expression changes, as well as identification of those genes that show statistically significant differential expression in the sample subgroups discovered. The options implemented in the present work are detailed in the following subsections.

Class-preserving resampling. Consider a case of two distinct clinical classes, denoted by $A$ and $B$, and hidden sample subgroups inside these classes, for example, disease subtypes within the patient category. The rationale of resampling phase is to cater for the full spectrum of within-class differences between these two sample classes, while making the identification results more robust against unknown sample subgroups or labeling errors present in the dataset. In the present work, pairwise sample comparisons between the classes $A$ and $B$ were used in the resampling phase, although other schema, such as class-preserving bootstrapping, could be used instead. To further decrease the sensitivity of the detected subgroups to artefacts in the expression intensities, we used a rank-based scoring of the genes in each individual sample. The benefits of using ranks instead of the intensity values in the detection of differential expression have been demonstrated, for instance, in the rank product method (Breitling et al., 2004).

More formally, let us denote by $\mathbf{x}_{i}=\left(x_{i 1}, x_{i 2}, \ldots, x_{i k}\right)^{\mathrm{T}}$ and $\mathbf{y}_{j}=\left(y_{j 1}, y_{j 2}, \ldots, y_{j k}\right)^{\mathrm{T}}$ the preprocessed logarithmic expression values of samples $i=1,2, \ldots, m$ and $j=1,2, \ldots, n$ in the classes $A$ and $B$, respectively, where $k$ is the number of genes probed. The expression vector $\mathbf{x}_{i}$ of the sample $i$ in the class $A$ was first compared to each of the vectors in the class $B$, and the $n$ vectors resulting from these comparisons were then aggregated for the sample $i$. For ranking the genes in such pairwise comparisons, we chose to use the fold-change statistic, and aggregation of the individual-specific rank vectors was done on the basis of the geometric mean of the gene ranks. More specifically, the score vector $\mathbf{s}_{i}$ for the sample $i$ in the class $A$ was calculated as

$$
\mathbf{s}_{i}=\left(\prod_{j=1}^{n} \rho\left(\mathbf{x}_{i}-\mathbf{y}_{j}\right)\right)^{1 / n}
$$

where $\rho$ is the sample rank function. This vector-valued function assigns a rank for each element in the fold-change vector according their increasing order of magnitude; thus, the rank of the smallest fold-change value equals 1 , the rank of the second smallest value equals 2, etc., and finally, the rank of the largest value equals the number of nonmissing values in the vector. The missing values were preserved in the rank vectors and average ranks were used in case of ties. The product of the vectors and their involution in the equation (1) were calculated element-wise. Similarly, the score vector $\mathbf{t}_{j}$ for the sample $j$ in the class $B$ was calculated as

$$
\mathbf{t}_{j}=\left(\prod_{i=1}^{m} \rho\left(\mathbf{y}_{j}-\mathbf{x}_{i}\right)\right)^{1 / m} .
$$

From the score vectors $\mathbf{s}_{i}$ and $\mathbf{t}_{j}$ we constructed a $k$-by- $(n+m)$ score matrix $\left(\mathbf{s}_{1} \mathbf{s}_{2} \ldots \mathbf{s}_{m} \mathbf{t}_{1} \mathbf{t}_{2} \ldots \mathbf{t}_{n}\right)$. This score matrix resembles the original expression value matrix and can be used as its robust alternative in the subsequent data analysis steps, such as discovery of sample subgroups and detection of subgroupspecific differential expression.

Subgroup detection. In the present work, the sample subgroups within the classes $A$ and $B$ were visualized and discovered using the divisive hierarchical clustering algorithm implemented in the $\mathrm{R}$ package cluster (Kaufman and Rousseeuw, 1990). The particular subgroups for further analysis can be then extracted either visually or automatically from the resulting hierarchical tree (dendrogram). Our choice 


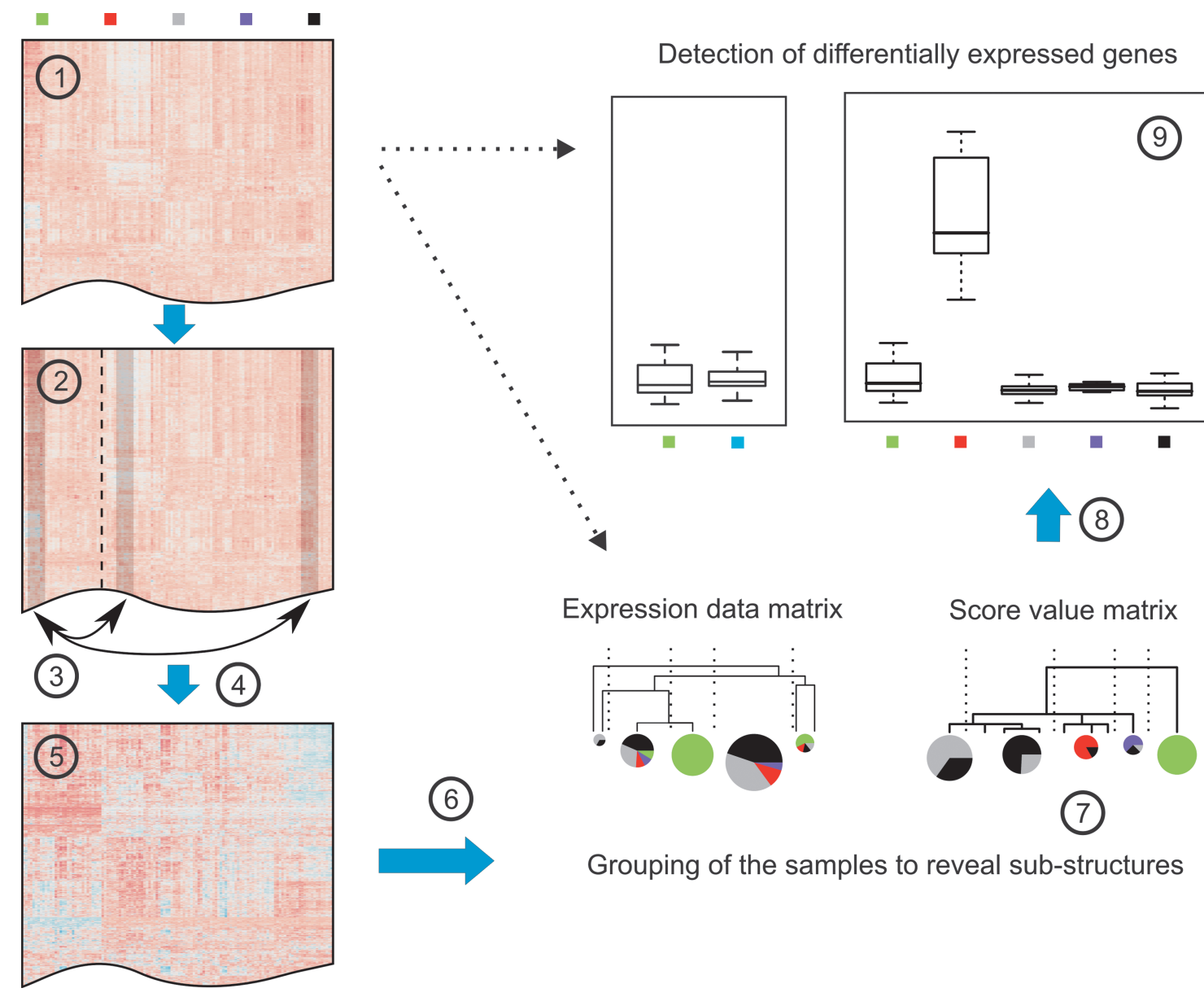

FIG. 1. Schematic illustration of the analysis workflow. The steps of the generic ReScore framework, with optional choices for each step listed after the numbers (the options used in the current work are in italic font). The blue arrows depict the proposed workflow; the dotted arrows are the conventional approaches. The different steps are illustrated using real data from the ALL material; colors indicate the sample subgroups (green, T-lineage NEG; red, B-lineage ALL1/AF4; gray, B-BCR/ABL; magenta, B-E2A/PBX1; black, B-NEG; and blue, B-Summary sample). (1) The inputs of the procedure are a preprocessed expression data matrix, in which the rows represent the genes (or probesets) and the columns stand for the different samples (arrays), and the sample labels of the given classes to be compared (here T- and B-lineages). Possible preprocessing options include log-transformation, between-array normalization, and variance-stabilization. (2) At the core of the procedure is the class-preserving resampling phase, in which the samples across the given classes are iteratively compared (the class border is indicated by the dotted line). Possible resampling schema include paired samples, bootstrap sampling, and random subsets. (3) In each subsample comparison, the genes are ranked according to the differential expression between the sample subsets selected in the current resampling iteration. Possible comparison statistics include fold-change, $t$-statistic or its modifications. (4) After a selected number of iterations, the rank-based gene scorings of each sample are aggregated on the basis of all those comparisons in which the particular sample was present. Possible rank aggregation methods include the geometric mean or the Borda count. (5) The output of the resampling-procedure is a transformed score value matrix, in which the individual gene scores for each sample carries information on the systematic differences across the classes, while being robust against the unknown sample subgroups or labeling errors present in the dataset. (6) Calculating the dissimilarities between the sample pairs on the basis of their aggregated gene score vectors. Possible distance measures include Pearson correlation, Euclidean distance, or the p-norm. (7) Grouping of the samples and visual quality control can be done by means of the output of the selected clustering method, such as hierarchical clustering, partitioning around medoids, or model-based clustering. The transformed score matrix facilitate grouping of the clinically related samples together (the right dendrogram), compared with completely unsupervised group discovery (the left dendrogram). The size and the colors of the pie charts indicate the number of samples in the cluster and their classification into the five subgroups. (8) The extraction of the particular sample subgroups for further analysis can be done either visually or automatically. (9) The final step is the detection of significant genes that can distinguish the sample subgroups. Statistical significance can be assessed either by controlling the family-wise error rates ( $p$-values), or the false discovery rate (FDR). With the help of the resampling-based procedure, one can reveal significant differences specific to the hidden sample subgroups (the right box-plot), which would be missed without the sample grouping phase (the left box-plot), because of the confounding variability from the three other hidden subgroups. 
was the automatic Dynamic Hybrid Cut algorithm implemented in the R-library dynamicTreeCut (Langfelder et al., 2008). This bottom-up tree-cutting algorithm defines the clusters in two subsequent steps. First, a set of preliminary clusters are identified as branches of the dendrogram that satisfy a number of compactness constraints while maintaining the minimum number of cluster members (set here to five). In the second step, all the remaining samples are assigned to their closest cluster (Langfelder et al., 2008). This dynamic technique is well suited for automation, capable of identifying also nested clusters, and it also provides more flexibility than the commonly used fixed height cutoff method that may exhibit suboptimal performance on complicated dendrograms.

As a dissimilarity measure for the pairs of samples in the cluster division process, we used the so-called $p$-norm distance (i.e., Minkowski distance of order $p$ ). For the score vectors $\mathbf{s}_{i}$ and $\mathbf{t}_{j}$, the $p$-norm distance is defined as

$$
\left\|\mathbf{s}_{i}-\mathbf{t}_{j}\right\|_{p}=\left(\sum_{l=1}^{k}\left|s_{i l}-t_{j l}\right|^{p}\right)^{1 / p},
$$

where $p$ is a nonnegative integer. The two well-known special cases of the $p$-norm distance are Manhattan distance $(p=1)$ and Euclidean distance $(p=2)$. When $p$ approaches infinity, the value of the $p$-norm distance approaches the maximum of the absolute distances of the components of the two vectors (so-called maximum norm). Consequently, the larger the $p$ the larger is the effect of those genes whose scores differ the most. This property of the $p$-norm makes it ideal for discovering hidden subgroup structures in the data that are characterized by coherent expression changes often in a relatively small number of genes only.

Gene identification. Once the sample subsets have been extracted, the final phase of the procedure involves identification of those genes that are differentially expressed between the subsets discovered in $A$ and $B$. Because we used pairwise rankings of the fold-change differences in the preset work, the gene identification could be carried out similarly as in the rank product method (Breitling et al., 2004). The standard application of the rank product method, in fact, represents a special case of the ReScore procedure when no sample subgroups are detected before gene detection. To use the implementation of the rank product as a part of our procedure in the detection of both up- and downregulated genes, we also calculated score vector $\mathbf{s}^{\prime}{ }_{j}$ for each sample in the class $A$ by replacing the rank function $\rho$ in equation (1) with the reversed rank function $\rho^{\prime}$, which ranks the fold-change vectors in decreasing order.

The statistical significance of the subgroup-specific marker genes was controlled by means of the false discovery rate (FDR) levels, that is, the expected proportion of false detections among the set of all detections (Benjamini and Hochberg, 1995; Storey, 2002; Tsai et al., 2003). In the present work, the FDR-level of a particular gene (the so-called $q$-value) was estimated by permuting the sample labels 100 times and then calculating the average number of genes in such random experiments that give the same or better rank product than the observed one (the expected proportion of false detections), divided by the rank of the gene in the original list (the number of detections). Because the statistical testing is effectively the same as in the original rank product method, once the sample subgroups have been detected, we used the permutationbased FDR-estimation method implemented in the RankProd R-package (Hong et al., 2006). FDR-estimates larger than one were truncated to unity.

\section{Data sets and evaluation procedure}

We tested and compared our procedure on three different real microarray data sets in which molecularly, histologically or pathologically defined sample subsets have been established. These sample subsets were withheld from the data analysis when evaluating the ReScore procedure against the conventional approaches; standard testing of differential expression and unsupervised clustering of the samples (the two dotted arrows in Fig. 1). Here, we detail the benefits of our procedure on two of the datasets (ALL and Endometriosis materials), while the grouping accuracy results on the breast cancer dataset are briefly given in Table 1. Because the clinical datasets are bound to contain also additional hidden sample subgroups other than those used in the evaluation procedure, perfect classification of the samples using the expression data alone is nearly impossible in practice. Hence, these datasets

Table 1. Sample Grouping Accuracies in the Breast CANCER DATA

\begin{tabular}{llll}
\hline \multirow{2}{*}{ Hidden subgroups } & \multicolumn{3}{c}{ Known sample classes } \\
\cline { 2 - 4 } Grade & Grade & Metastasis & ER \\
& Intensity & 0.52170 & 0.70965 \\
& Filtered & 0.54601 & 0.75934 \\
Metastasis & ReScore & 0.70725 & 0.77136 \\
& 0.52170 & Intensity & 0.60955 \\
ER & 0.54601 & Filtered & 0.63677 \\
& 0.72192 & ReScore & 0.70440 \\
& 0.70965 & 0.60955 & Intensity \\
& 0.75934 & 0.63677 & Filtered \\
& 0.93723 & 0.82256 & ReScore \\
\hline
\end{tabular}

The breast cancer dataset of van't Veer et al. (2002) was processed using either a standard hierarchical clustering (HC) method (divisive algorithm with correlation as dissimilarity measure) or with the ReScore procedure (the order $p=37$ was selected on the basis of the number of genes in the unfiltered data). Three clinical parameters, namely, the histological grade of the tumor, the metastasis status after 5 years following the diagnosis, and the estrogen receptor (ER) status of the tumor, were used as grouping parameters, in turn, to determine either the known sample classes under the comparison (rows) or the hidden sample subgroups considered as unknown (columns). In each matrix entry, the three values correspond to the $F$ values calculated using either the $\mathrm{HC}$ with or without gene filtering or using the ReScore procedure on the unfiltered dataset. Filtering of the genes from the original 24,481 to 4,348 was carried out using the same rules as in the original work (that is, requiring at least a twofold difference and a $p$-value of $<0.01$ in more than five tumors). In the comparisons involving the metastasis status, 20 out of the total 98 patients who carried a BRCA germline mutation were excluded because they did not take part in the metastasis follow-up evaluation. In each of the cases, the sample grouping with the ReScore procedure on the unfiltered data provided improved association with the groupings established by means of the clinical parameters, compared with those of the HC approaches. In general, the tumor ER-status was best captured on the expression data, but the ReScore procedure was able to reveal also the more subtle expression variation linked to the metastasis and grade status. 
provide a practical setting for evaluating the performance of the different approaches.

ALL material. The acute lymphocytic leukemia (ALL) material consisted of samples from 128 adult patients with newly diagnosed ALL (Chiaretti et al., 2005). Phenotypic classification between T-cell and B-cell lineages was determined by conventional immunophenotypic analysis of cell surface markers. The B-lineage ALL group contains three distinct molecular rearrangement groups, confirmed by molecular diagnostic studies, namely, BCR/ABL, ALL1/AF4, and E2A/PBX1. These gene rearrangements were identified in 37,10 , and 5 cases, respectively. There were also 42 patients in the B-ALL group and 32 in the T-ALL group without evidence of known molecular rearrangements (labeled as NEG).

The samples were hybridized to the Affymetrix U95Av2 GeneChip, which contains 12,625 probe sets and the data were normalized by RMA (Irizarry et al., 2003). It was shown in the original work using the standard hierarchical clustering, that the expression profiles of the T-lineage cases were relatively homogeneous, when compared to the B-lineage cases (Chiaretti et al., 2005). Especially, the E2A/PBX1 and ALL1/AF4 samples formed distinct expression clusters, while the $\mathrm{BCR} / \mathrm{ABL}$ group is more heterogeneous and similar to the NEG group. In the evaluation, we considered the two phenotypic classes (T- and B-lineages) as the known sample categories, and the four subtypes of genotypic abnormalities (E2A/PBX1, ALL1/AF4, BCR/ABL, and NEG) as the hidden subgroups.

Endometriosis material. Endometriosis is a common medical condition characterized by the presence of functional endometrial glands and stroma in ectopic locations outside the uterine cavity, typically on the peritoneum, in the ovary or deeply infiltrated in the organs in the peritoneal cavity. The prevalence of endometriosis is roughly $40 \%$ in women with severe menstrual pain or infertility or both (Giudice and Kao, 2004). Our endometriosis material contained a total of 306 tissue samples, 72 of which were peritoneal and 78 deep endometriotic lesions, as well as 52 healthy peritoneum and 104 endometrium specimens, removed at surgical operation.

The tissue samples were hybridized to the Illumina Human 6 V2 arrays, which contains 48,701 probe sets. The raw intensity data were first extracted using the Illumina BeadStudio Gene Expression Module, then quantile-normalized using the lumi Bioconductor package (Du et al., 2008), and finally log-transformed. In the evaluation, the patient class consisted of three tissue- and lesion-specific sample subgroups, namely, the peritoneum samples of the patients as well as peritoneal and deep endometriotic lesions. The two hidden subgroups within the control class were the peritoneum samples of the healthy control subjects as well as the endometrium samples.

Synthetic subgroups. A comprehensive testing of the properties of the ReScore procedure was initially performed on real data-based simulations, in which blocks of samples and genes were randomly shuffled in part of the ALL dataset, while preserving the inherent distinction between the B- and T-lineages. Such synthetic subgroups structures allowed us to systematically evaluate its behavior in response to various factors, such as the number and sizes of the blocks generated and the effect of the filtering, in a relatively controlled setting on a real clinical material. The genes for the simulation purposes were first selected by following the filtering criteria similar to those in the original work (Chiaretti et al., 2005): the expression values must be above $\log _{2}(100)$ at least in $20 \%$ of the samples, and the interquartile range across the samples must be at least 0.4 . The resulting data matrix consisting of 3,020 genes and 128 samples was then two-way clustered (Chiaretti et al., 2005). On the grounds of visual inspection of the heat map, we chose the most homogeneous cases from the two categories, including 20 and 31 samples from the B- and T-lineages, respectively. To assure that the selected blocks were as homogeneous as possible, we further sorted the genes according to group-wise $t$-test and the samples using hierarchical clustering.

Synthetic subgroups were generated by choosing randomly blocks of samples and genes within the 3,020-by-51 data matrix (Fig. 2). The sample-coordinate of each block was restricted such that the whole block fits inside one class only (either B or T), and the gene-coordinate was restricted to the upper one-third of the matrix to guarantee that the blocks contained genes that show differential expression between the

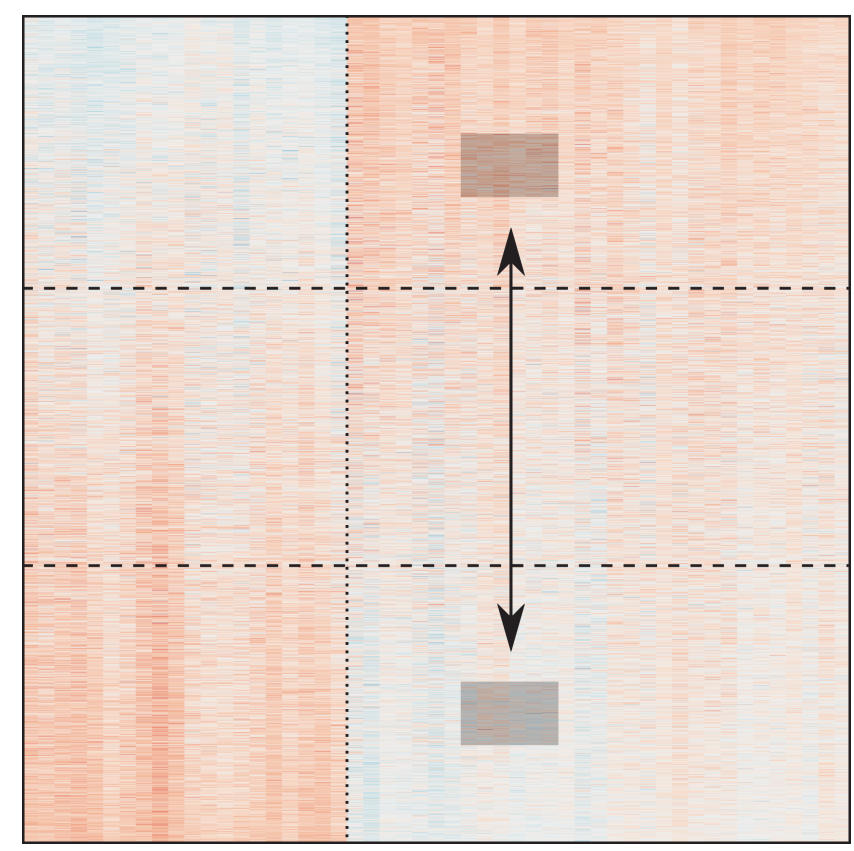

FIG. 2. Generating synthetic subgroups in the ALL material. In this 3020-by-51 submatrix, the genes (rows) were ordered according to group-wise $t$-test and the samples (columns) using hierarchical clustering to guarantee maximal local homogeneity. Synthetic subgroups were generated by choosing randomly blocks of samples and genes within the data matrix (the upper gray box). The sample-coordinate of each block was restricted such that the whole block fits inside one class only (either B or T, the horizontal dotted line), and to guarantee that the blocks contained genes that show differential expression between the two lineages, the genecoordinate was restricted to the upper one-third of the matrix (the vertical dotted line). Another block of the same size was then randomly selected at the same sample-coordinate but from the lower one-third of the genes (the lower gray box), and the expression values of these two blocks were swapped. 
two lineages. Another random block of the same size was then randomly selected at the same sample-coordinate but from the lower one-third of the genes, and the expression values of these two blocks were swapped. Such block-wise permutations result in distinct sample subgroups with coherent expression changes in relatively homogeneous background. To test the effect of adding genes that are noninformative for the classification between the T- and B-lineages, 9,605 noise genes were added at the bottom of the data matrix. The noise genes were constructed by randomly permuting the sample labels of those 9,605 genes that were discarded in the initial gene selection. In each simulation run, we generated one or two subgroups of varying sizes within the T- or B-class. For each block size, the simulations were repeated for 100 random block locations.

Evaluation measures. The groupings of the samples were evaluated with respect to the a priori established sample subsets using the F-measure (van Rijsbergen, 1979). The $F$-measure for a given sample class $i$ and a detected sample cluster $j$ is defined as

$$
F(i, j)=\frac{2 P(i, j) R(i, j)}{P(i, j)+R(i, j)},
$$

where $P$ and $R$ are the precision and recall between the sample sets $i$ and $j$, respectively. Although the $F$-measure has traditionally been applied to partitional clustering, it can also be used to evaluate entire hierarchical clustering tree by traversing it recursively and considering each subtree as a cluster (Seo et al., 2004). Like all external cluster validation measures, the observed value of $F$ should be normalized to account for the unwanted effects due to, for example, the number and size of the given groups and differences between datasets (Handl et al., 2005). In contrast to partitional clustering, however, simple permutation of the original labels of the samples is not sufficient in the hierarchical clustering case because the overall structure of the resulting dendrogram does not depend on the labels.

We therefore developed novel metrics to compare the relative performance of the different sample clustering results using the F-measure. To evaluate the methods with respect to random clustering of the same samples, we permutated the distance values of the original dissimilarity matrix, and thereby generated random dendrograms of the same data. To preserve the phenotypic distinction, for example, the B- and T-lineages in the ALL dataset, we permutated the different segments of the dissimilarity matrix separately (Fig. 3A). Such permutation scheme generally preserves the given classes, while allowing the samples within these clusters to mix. Therefore, it provides a reasonable estimate of the range of $F$-values that could be obtained by chance alone.

In theory, the $F$-measure can take values between 0 and 1 . In practice, however, the range of $F$-values obtained for a given external partitioning is more limited. To compare the methods with respect to practical minimum and maximum values, data-specific upper and lower bounds for the $F$-measure were defined in the ALL dataset. We calculated the practical minimum by assuming that the T-and B-lineage samples form distinct clusters (Fig. 3B). When calculating the practical maximum, we additionally assumed that the ALL1/AF4 and E2A/PBX1 samples cluster in different subtrees, whereas the $\mathrm{BCR} / \mathrm{ABL}$ and NEG samples cluster together in the same subtree (Fig. 3C), according to the earlier results (Chiaretti et al., 2005). Therefore, $F_{\min }=0.64500$ and $F_{\max }=0.79175$ in this material.

\section{Results}

\section{Synthetic subgroups in the ALL dataset}

We initially evaluated the properties of the ReScore procedure using artificially generated subgroup block structures in the ALL material. Here, we focus on those factors that affect the optimal order of the $p$-norm when distinguishing the
A

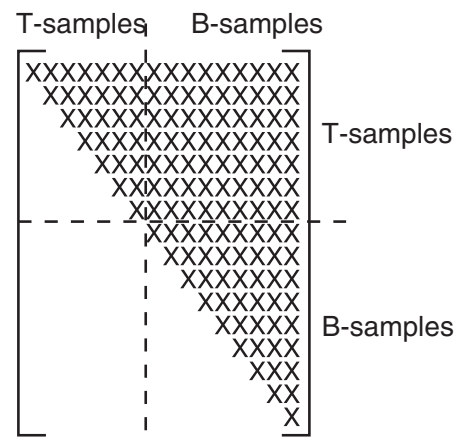

B

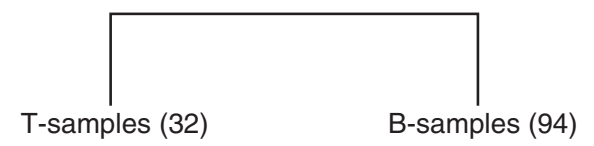

C

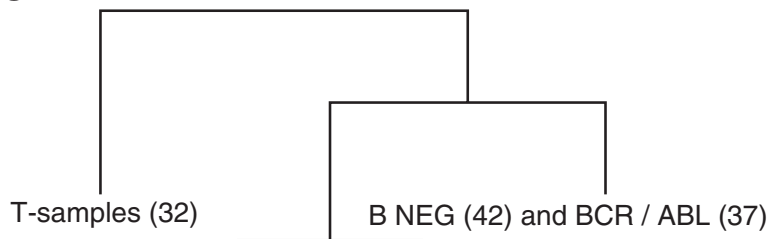

ALA

ALL1 / AF4 (10) E2A / PBX1 (5)

FIG. 3. Permutation of the dissimilarity matrix. (A) The three segments in the upper triangular of the dissimilarity matrix of the ALL dataset were permuted separately to preserve the given classes. After each permutation, the upper triangular was copied to the lower triangular. (B) The practical of minimum of the F-measure was calculated by assuming that the T- and B-lineage samples form completely distinct clusters. (C) The practical maximum was calculated by assuming that the B-lineage molecular subgroups (NEG and BCR/ABL) do not separate but the two other subgroups (ALL1/AF4 and E2A/PBX1) form distinct clusters. 
underlying sample subgroups using hierarchical clustering. The performance of the $p$-norm as a function of $p$ was compared to that of the Pearson correlation (the zero $y$-level in Fig. 4$)$ and the Euclidean distance $(p=2)$. As expected, the number of genes in the dataset had the biggest influence on the behavior of the p-norm (Fig. 4 first column vs. other columns); the more genes there are probed, the bigger the $p$ should be. Also, the number of subgroups present in the data increased the appropriate value of $p$, and also improved the relative performance of the $p$-norm compared to the correlation especially in the smaller dataset (Fig. 4A vs. B). The number of blocks increased the $p$-value also after adding noninformative genes to the data, albeit the effect was smaller in the unfiltered dataset (Fig. $4 \mathrm{C}$ vs. D).

Once the number of genes and blocks was fixed, whether the subgroups were generated in the T- or B-classes did not have a substantial effect on the optimal $p$-value (Fig. 4D vs. E). The ability how accurately the procedure could distinguish the hidden subgroups from the data was, however, different in these two cases. This was rather expected, as the T- and B-classes present with different degree of interindividual variability. Similarly, although the dimension of the blocks affected their identification accuracy, the relative performance in terms of the optimal $p$-value was surprisingly robust against the number samples and genes in the blocks (Fig. 4E vs. F). In some cases, there was a slight decrease in the performance after $p=30$, especially when larger block sizes were generated (Fig. 4F). In practice, however, the hidden sample subgroups may be relatively small in their size, which supports the usage of larger $p$-values in general.

The comprehensive real data-based simulations in the various settings showed that one can easily find such values of $p$ that the performance of the corresponding $p$-norm outperforms the commonly used distance measures. Moreover, the scoring system is not very sensitive to this choice, but a wide range of different $p$-values showed systematically better behavior than the correlation and Euclidean distances. The results indicated that -as a rule of thumb - the $p$-value depends fairly linearly on the number of genes probed, but is more or less independent on the many other factors such as the number of samples and genes in the subgroup. Increasing the number of subgroups makes the dataset more heterogeneous and thus affects the choice of the $p$-value. Because the number of sample subsets in unknown in practice, it is generally advisable to use larger values of $p$ in real applications. In the following applications, we therefore chose on the basis of the linearity, $p=15,30$, and 45 for datasets with 3,020, 12,625 , and 48,701 genes, respectively.

\section{Application to the full ALL dataset}

Next we investigated how accurately the procedure could distinguish the a priori established four molecular subtypes in the B-lineage (E2A/PBX1, ALL1/AF4, BCR/ABL, and NEG), compared to the conventional hierarchical clustering approaches. When applied to the dataset containing the 3,020 filtered genes, the ReScore was the most accurate in discovering these subgroups (Table 2). Even if the differences in the $F$-values were not marked, comparison against the practical minimum and maximum values provided already some support for the relative improvements gained over the standard hierarchical clustering of the samples. The relative differences between the five grouping approaches became much more evident when all of the 12,625 genes were used (Table 2). In this case, the performance of the correlation and Euclideanbased clustering was, in fact, similar to that of random clustering, whereas ReScore could improve its performance on the unfiltered data. Investigation of the dendrograms obtained
A
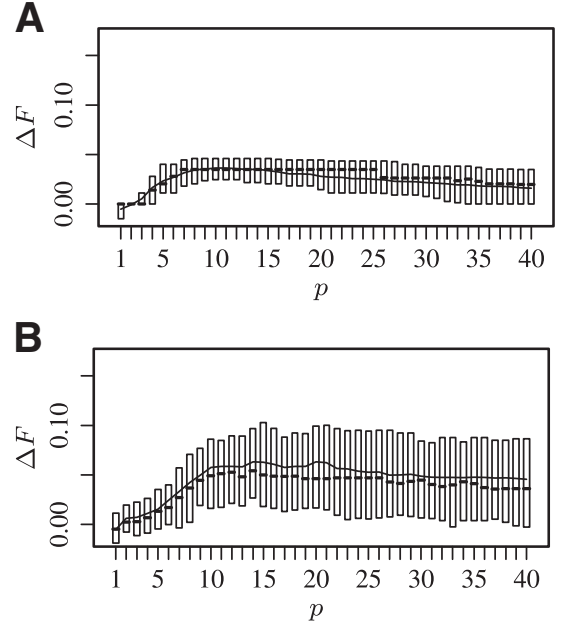
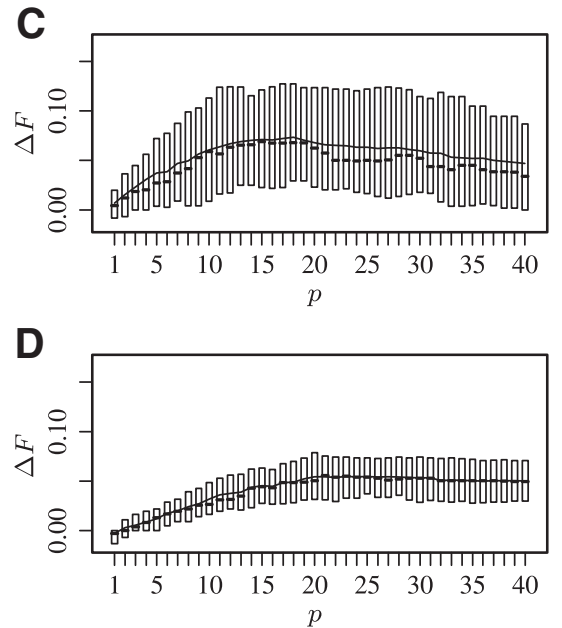

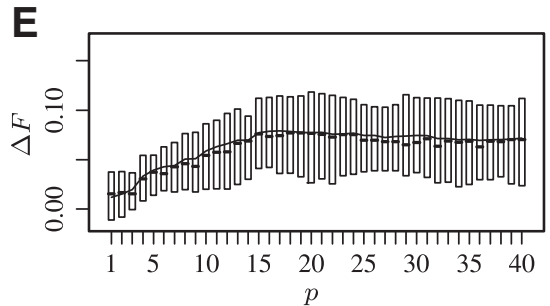

$\mathbf{F}$

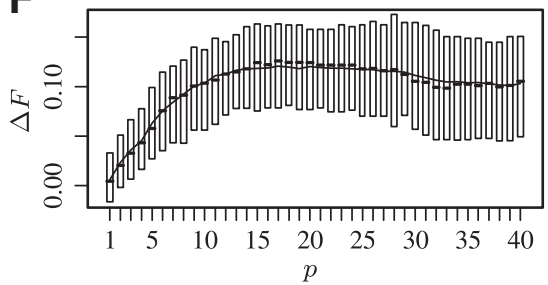

FIG. 4. Evaluating the performance of the $p$-norm. The effect of the order $p(x$-axis) on the identification of the synthetic subgroups was evaluated as a relative pairwise difference in the $F$-measure $(\Delta F)$ between the $p$-norm and the correlation ( $y$-axis). The similarity measures were applied to the score matrix. Various numbers and sizes of blocks of samples and genes were generated in the T- or B-lineages of the filtered and unfiltered ALL dataset: (A) one block (4 samples, 50 genes) in B, 3020 genes. (B) Two blocks (6 and 10 samples, 25 genes) in T, 3,020 genes. (C) One block (10 samples, 25 genes) in T, 12,625 genes. (D) Two blocks (4 and 6 samples, 25 genes) in B, 12,625 genes. (E) Two blocks (6 and 10 samples, 25 genes) in T, $12 \mathrm{~m} 625$ genes. (F) Two blocks (10 and 10 samples, 50 genes) in T, 12,625 genes. The box plots show the median (bolded horizontal lines), mean (thin trace), and the interquartile range of $\Delta F$ (border lines). 
Table 2. Accuracy of the Sample Grouping Strategies with and without Gene Filtering

\begin{tabular}{lcccc}
\hline & \multicolumn{2}{c}{ Filtered (3020 genes) $^{2}$} & \multicolumn{2}{c}{ Unfiltered (12625 genes) } \\
\cline { 2 - 4 } Method & F-value $^{\mathrm{a}}$ & Normalized $^{\mathrm{b}}$ & F-value & Normalized \\
\hline ReScore procedure & 0.74258 & 0.66494 & 0.78614 & 0.96177 \\
Agglom HC (cor) $^{\mathrm{c}}$ & 0.69010 & 0.30733 & 0.65127 & 0.04273 \\
Agglom HC (euc) $^{\mathrm{d}}$ & 0.72023 & 0.51264 & 0.67605 & $-0.05090^{\mathrm{e}}$ \\
Divisive HC (cor) & 0.72961 & 0.57656 & 0.66332 & 0.21158 \\
Divisive HC (euc) & 0.64204 & $-0.02017^{\mathrm{e}}$ & 0.66380 & 0.12484 \\
Random grouping & 0.66581 & 0.14181 & & 0.12811 \\
\hline
\end{tabular}

${ }^{\text {a }}$ The ability to reveal the four a priori established sample subsets in the B-lineage.

${ }^{\mathrm{b}} F_{\text {normalized }}=\left(F-F_{\min }\right) /\left(F_{\max }-F_{\min }\right), F_{\min }=0.64500$ and $F_{\max }=0.79175$.

'Hierarchical clustering (HC) with Euclidean distance, or ${ }^{\mathrm{d}}$ one minus correlation on the preprocessed expression data.

${ }^{\mathrm{e}}$ Because the HC does not use the given class labels, its performance can be even lower than the practical minimum.

from the different approaches also revealed several marked differences in how the samples were grouped together (Figs. 5 and 6).

An important follow-up question concerned whether the enhanced sample clustering performance contributed also to more accurate identification of the disease-related genes, compared to the standard analysis of differential expression alone. We therefore automatically identified a subtree which, by and large, corresponded to one of the established disease subtypes (ALL1/AF4; Fig. 5). Even if the procedure included also two additional NEG samples, the majority of the top genes detected from this subgroup were those having most differential expression between the established ALL1/AF4 and T-class samples (Fig. 7 upper trace). In contrast, a large percentage of the ALL1/AF4-related genes were missed when the standard rank product method was applied to the B- and T-lineages as a whole (Fig. 7, lower trace). Similar behavior was observed also with other standard statistics (Fig. 8) and sample subsets (data not shown). These results demonstrate that the ReScore procedure can take full advantage of the unfiltered data when identifying the genes directly linked to a specific disease subtype, while not being very sensitive to the $100 \%$ accurate sample classification. The two extra NEG samples, in fact, could even be missed candidates for the ALL1/AF4 genotype.

The investigation of the top genes identified with the ReScore procedure revealed that 16 out of the 19 genes identified in the original study had FDR below 0.05 in our analysis

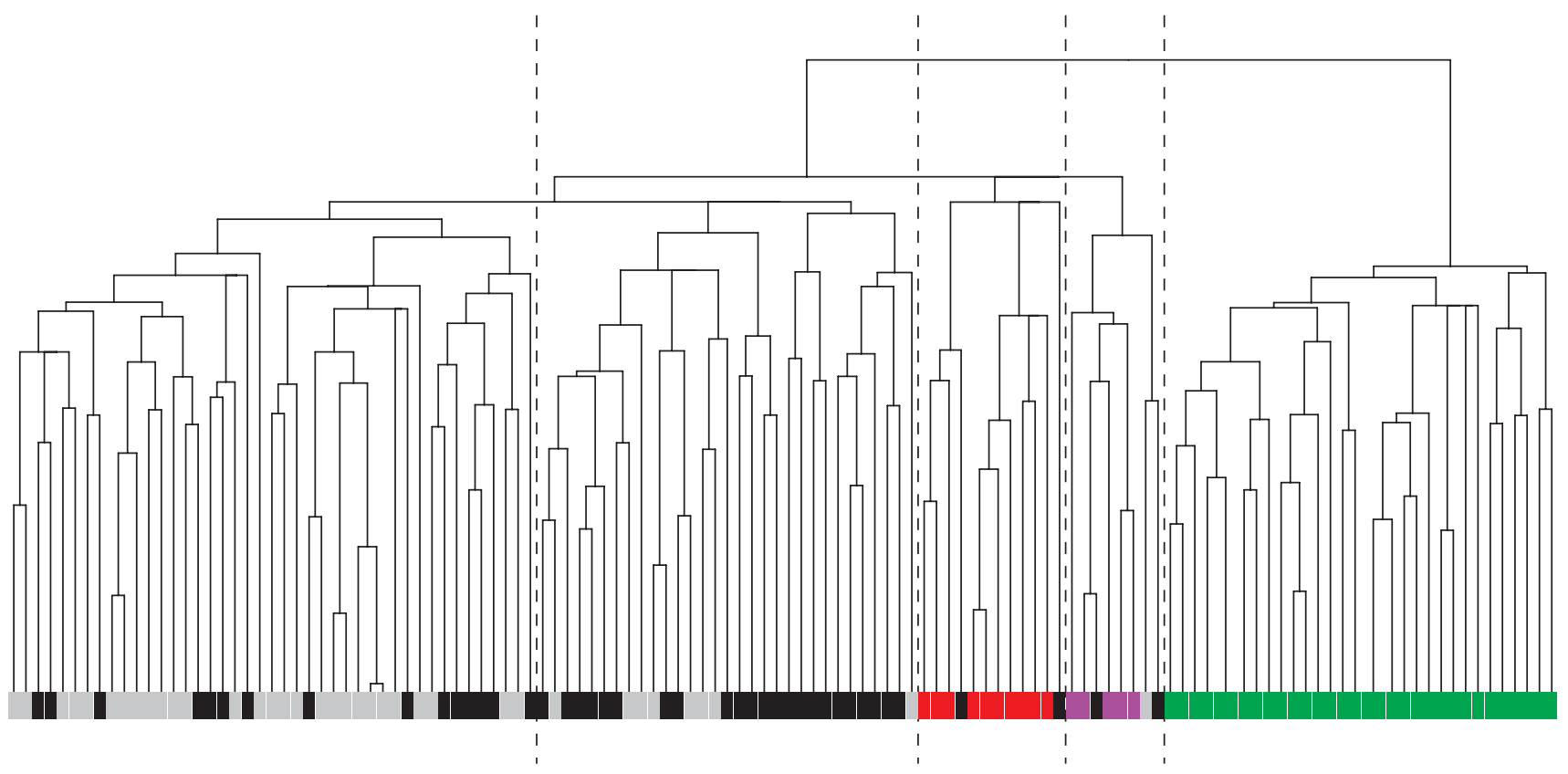

FIG. 5. Grouping of the ALL samples. The dendrogram was obtained by applying the ReScore procedure to the full ALL dataset. The heterogeneous B-lineage samples were identified as a separate cluster (the left subtree), in which the colors indicate the hidden sample subgroups (red, ALL1/AF4; magenta, E2A/PBX1; gray, BCR/ABL; and black, samples without known molecular rearrangements, NEG). The dashed lines show the five subgroups discovered with the Dynamic Hybrid cut method. The two most interesting molecular rearrangements in the B-lineage (ALL1/AF4 and E2A/PBX1) were clustered separately into distinct subtrees. The T-lineage NEG samples were also grouped perfectly together in the right-most subtree (the green color). For comparison, the grouping of the same samples with standard hierarchical clustering is shown in Figure 6. 


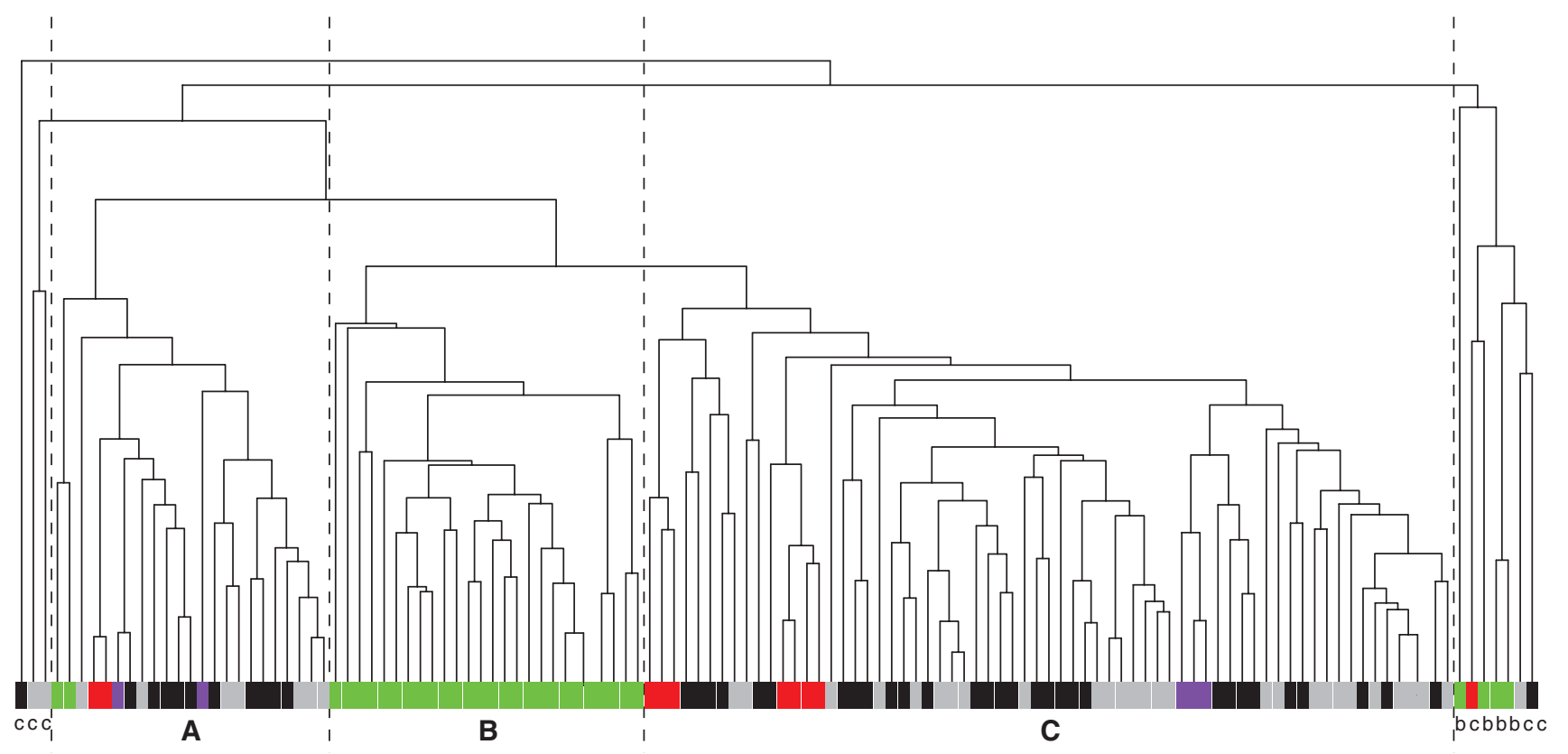

FIG. 6. Grouping of the ALL samples using standard hierarchical clustering approach. The full ALL dataset was clustered with agglomerative clustering algorithm using the 1 -correlation as the dissimilarity measure $(F=0.65127)$. The colors indicate the different sample subgroups (gray, BCR/ABL; black, B-lineage NEG; red, ALL1/AF4; magenta, E2A/PBX1; green, T-lineage NEG). The vertical dashed lines show the three subgroups discovered with the Dynamic Hybrid Cut method (labeled as A, B, and C). The three left-most and the seven right-most samples were left unassigned in the first step when the algorithm used the dendrogram structure only. The Dynamic Hybrid Cut algorithm includes an optional second step that can assign all the unassigned samples to the clusters detected in the first step according to the dissimilarities between the samples. The assignments made in the second step are indicated by lowercase letters below the leaves). However, even such advanced cluster detection method could not identify the two interesting molecular rearrangements groups (ALL1/AF4 and E2A/PBX1), but these samples were scattered around the dendrogram, which prevents their subsequent analysis such as identification of subgroup-specific genes (the ReScore-based dendrogram is shown in Fig. 5).

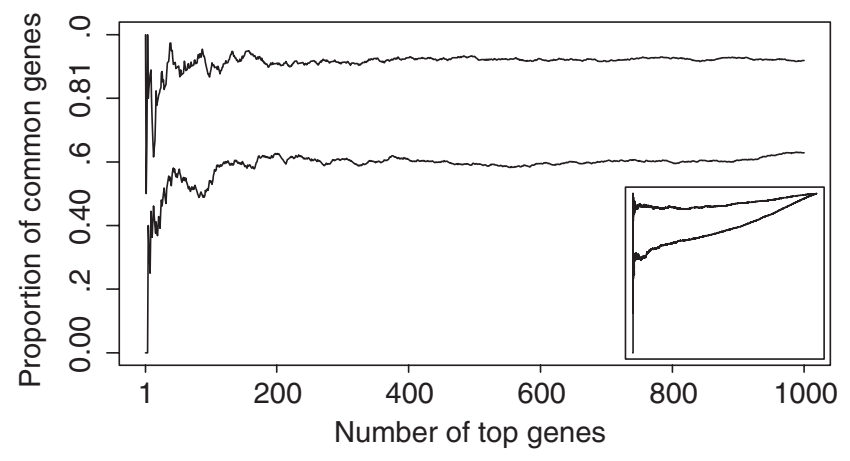

FIG. 7. Overlap in the ALL1/AF4-specific genes. The rank product method was used to rank the genes on the basis of differential expression when the T-class samples were compared with different subsets of the B-class samples. The upper trace: the overlap in the top genes between the established ALL1/AF4-cases and the ALL1/AF4-specific sample subset detected with the ReScore procedure; The lower trace: the overlap in the top genes between the detected ALL1/AF4-specific subgroup and all of the B-lineage samples using the standard rank product method. The inset shows the overall overlap profiles when the sizes of the top lists went all the way to 12,625 genes.
(Table 3). The PALLD and DIAPH2 genes were not considered significant in our analysis because we did not directly compare the subgroups of B-lineage to each other like was done in the original study (Chiaretti et al., 2005), but specifically focused on the differences between the ALL1/AF4 and T-samples. The expression values revealed that in both of these cases, the difference between ALL1/AF4 and T-groups was smaller than the differences within the B-ALL subgroups (Fig. 9). Even when using only the established ALL1/AF4 samples, these two genes were not significant (FDRs were 0.0839 and 0.2556 respectively), confirming that they are not in fact ALL1/AF4-specific genes. The third gene that was slightly outside the ReScore top list was NPTX2 (FDR = $0.0568)$, which was considered significant in the established ALL1/AF4 subgroup (FDR =0.0137). However, its rank was rather high (480th largest difference), and hence, it is not among the most promising candidate genes. In addition to these three genes, many other original findings would have been missed if the gene identification were carried out without performing the sample grouping phase (Table 3 ). In these cases, the resampling-based procedure could effectively cope with the expression variability resulting from the three confounding subgroups not under investigation (two example cases are shown in Fig. 10).

Finally, we determined the statistically significant pathway sets among the top genes from the ReScore procedure using the gene set enrichment analysis (GSEA) (Mootha et al., 2003; 
A

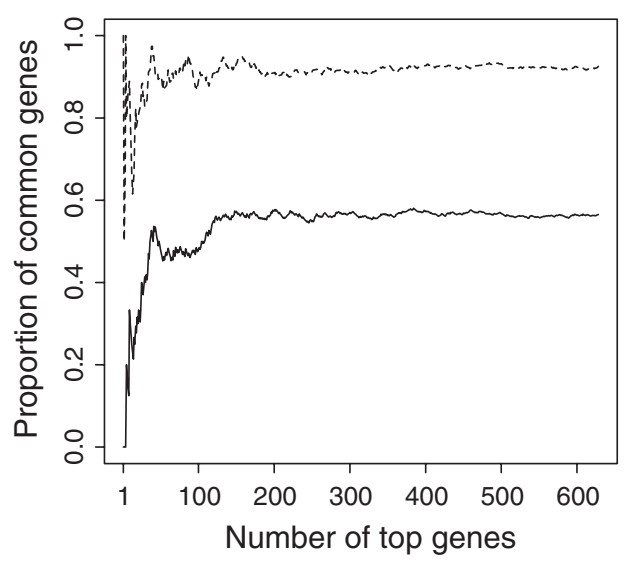

B

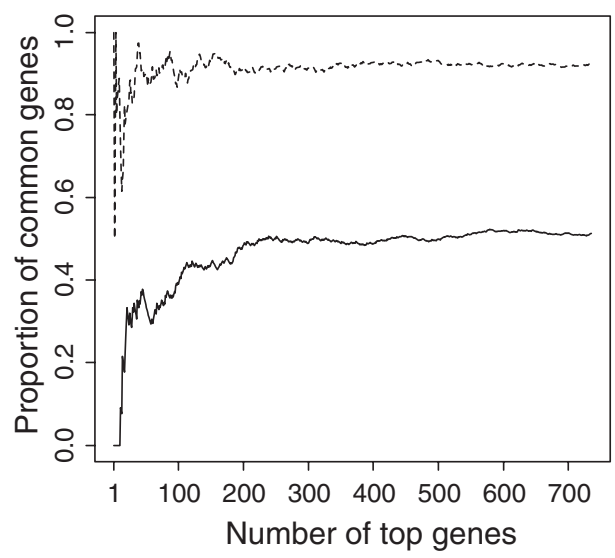

FIG. 8. Overlap in the ALL1/AF4-specific genes. The genes were ranked on the basis of differential expression when the samples in the T-class were compared with different subsets of the B-samples. The overlap in the y-axis is calculated with respect to the top genes between the established ALL1/AF4-cases and all the B-lineage samples using either the rank product method (A) or the standard $t$-test (B). The number of the top genes in the $\mathrm{x}$-axis goes through all the significant genes (the right-most gene in both panels has the FDR level of 0.05). These overlaps remain at a substantially lower level than when using the ReScore procedure (the dashed traces are from Fig. 7).

Subramanian et al., 2005). As a result, we found out that the top genes detected by the procedure were enriched in several leukemia-related pathways (Table 4). Three of the seven pathways were directly linked to leukemia (gene sets a, c, and $\mathrm{g})$, and three other were associated with hematopoiesis (b, e, and $\mathrm{f}$ ). Hematopoiesis refers to production of blood cellular components in the bone marrow and abnormalities in this development can lead to various blood cell diseases including leukemia (Sachs, 1996). In addition, RHOBTB3, CAP2, and
DPYSL3 genes belonged to the computational cancer modules 1 and 2 from MSigDB (Subramanian et al., 2005), and to our knowledge, there is only one previous study that shows that CAP2 gene is upregulated in human cancer (Shibata et al., 2006). These findings indicate that the top genes from the ReScore procedure are linked together in biological pathways and processes related to cancer and leukemia, many of which remained unidentified on the basis of the original study (Chiaretti et al., 2005).

Table 3. Rankings of the ALL1/AF4-Related Genes with and without Sample Grouping

\begin{tabular}{|c|c|c|c|c|c|}
\hline \multirow[b]{2}{*}{ Gene name $^{\mathrm{a}}$} & \multirow{2}{*}{$\begin{array}{c}\text { Subgroup- } \\
\text { specificity rank }\end{array}$} & \multicolumn{2}{|c|}{ With sample grouping } & \multicolumn{2}{|c|}{ Without sample grouping } \\
\hline & & $\operatorname{Rank}^{\mathrm{c}}$ & $F D R$ & $\operatorname{Rank}^{\mathrm{d}}$ & $F D R$ \\
\hline HOXA9 & 2 & 63 & $<0.0001$ & 9734 & 1.0000 \\
\hline HOXA10 & 4 & 143 & $<0.0001$ & 11346 & 1.0000 \\
\hline VLDLR & - & 26 & $<0.0001$ & 1038 & 0.0003 \\
\hline MEIS1 & 1 & 36 & $<0.0001$ & 3224 & 0.9784 \\
\hline DPYSL3 & 7 & 209 & $<0.0001$ & 5769 & 1.0000 \\
\hline PALLD & 201 & 996 & 0.1919 & 12477 & 1.0000 \\
\hline PPM1H ${ }^{\mathrm{f}}$ & 3 & 120 & $<0.0001$ & 3263 & 1.0000 \\
\hline PROM1 & - & 3 & $<0.0001$ & 191 & $<0.0001$ \\
\hline CCNA1 & - & 32 & $<0.0001$ & 722 & $<0.0001$ \\
\hline LGALS1 & - & 17 & $<0.0001$ & 362 & $<0.0001$ \\
\hline PRSS12 & 33 & 410 & 0.002 & 6453 & 1.0000 \\
\hline NPTX2 & 112 & 731 & 0.0568 & 12363 & 1.0000 \\
\hline FUT4 & 52 & 526 & 0.0146 & 4268 & 1.0000 \\
\hline TBC1D8 & - & 84 & $<0.0001$ & 915 & 0.0001 \\
\hline DIAPH2 & 261 & 1128 & 0.2825 & 10626 & 1.0000 \\
\hline DAD1 & - & 74 & $<0.0001$ & 1059 & 0.0006 \\
\hline HOXA5 & 10 & 224 & $<0.0001$ & 8824 & 1.0000 \\
\hline RHOBTB3 & 5 & 154 & $<0.0001$ & 2483 & 0.4569 \\
\hline KIAA0125 & 45 & 481 & 0.0078 & 11496 & 1.0000 \\
\hline
\end{tabular}

${ }^{a}$ Genes are arranged in the same order as in the original article (Chiaretti et al., 2005).

${ }^{\mathrm{b}}$ Because the genes in the original study were identified using ANOVA across the four B-lineage subsets, we deleted those genes with FDR $<0.05$ in the T- and B-lineages comparison (dashes) to ensure that the final set of genes were truly ALL1/AF4-specific.

${ }^{c}$ From the ALL1/AF4-associated subset of samples detected using the ReScore procedure.

${ }^{\mathrm{d}}$ From the comparison between all of the samples across the B- and T-lineages using the standard rank product method. Two of the gene annotations were changed compared to the original study: ${ }^{e}$ PALLADIN; ${ }^{f}$ KIAA1157. 
PALLD

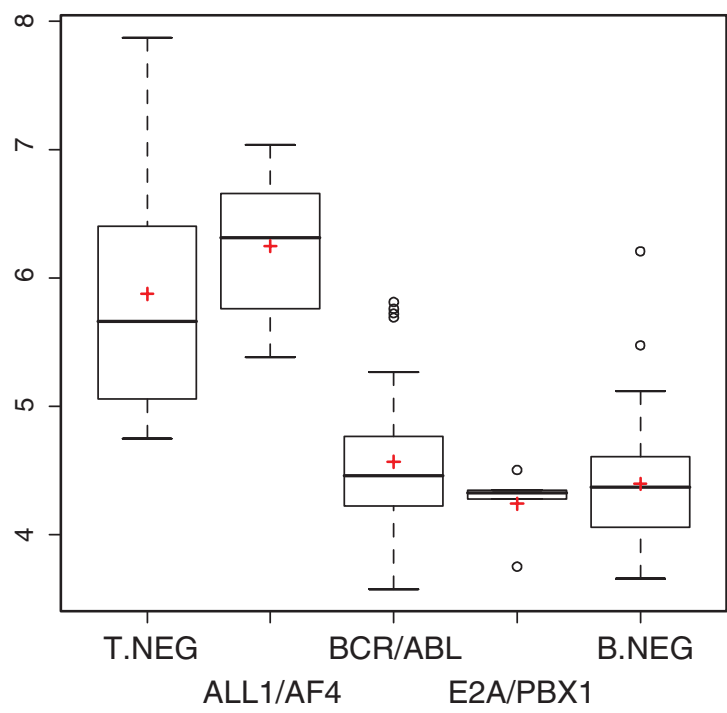

DIAPH2

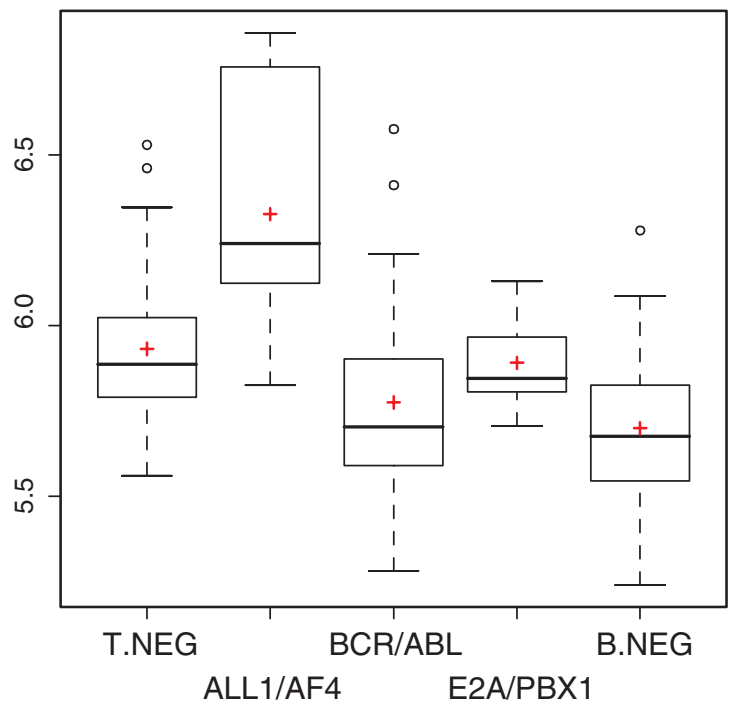

FIG. 9. Examples of the nonspecific ALL1/AF4-genes. Although there are significant differences across the four B-cell subgroups in the expression levels of PALLD and DIAPH2, which were identified in the original work (Chiaretti et al., 2005), the differences between the established ALL1/AF4 samples and the T-cell samples were nonsignificant (FDR was 0.0839 for PALLD and 0.2556 for DIAPH2). Therefore, these genes are not considered as truly ALL1/AF4-specific. In addition, these genes cannot distinguish between the T-cell and B-cell lineages (Table 3). The box plots show the median (horizontal lines), the mean (plus sign), the interquartile range (border lines), the robust range (error bars), and the outlying observations (circles) of the expression levels of the samples in the T-cell lineage (T.NEG), as well as in the four B-cell subgroups (ALL1/AF4, BCR/ABL, E2A/PBX1, and NEG).

\section{An independent case study of endometriosis}

The original reason for developing the resampling-based procedure was our in-house transcriptomics study of the endometriosis-related changes in gene expression. Here, it was used as an independent validation data set, showing that neither the procedure nor its parameters were overfitted to the ALL data set only. The ReScore procedure was more successful in discovering both the tissue- and disease-specific sample subgroups in the heterogeneous endometriosis data
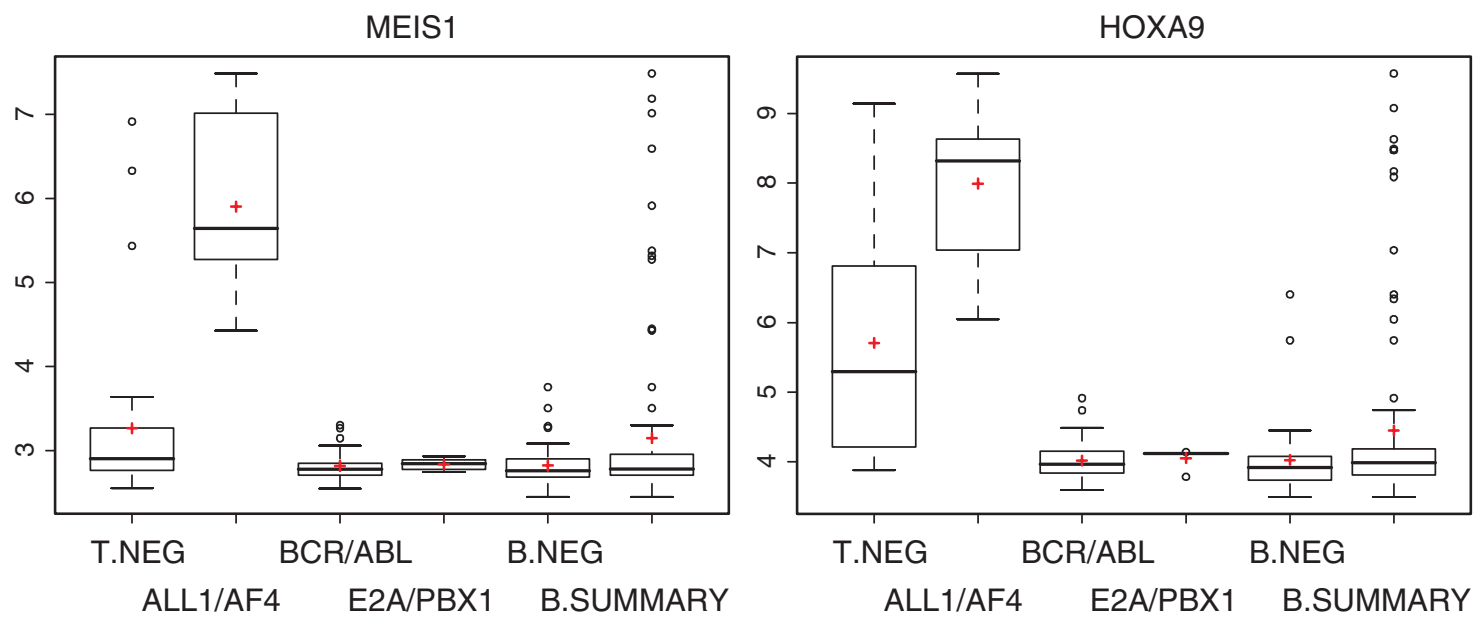

FIG. 10. Examples of the missed ALL1/AF4-specific genes. Even if there are significant differences between the ALL1/AF4 and the T-cell samples in the expression levels of MEIS1 and HOXA9, which were correctly identified with the ReScore procedure (the first two genes in Table 4) similarly as in the original work (Chiaretti et al., 2005), the other B-cell subgroups make the differences between the B- and T-classes highly nonsignificant (FDR was 0.9784 for MEIS1 and 1.276 for HOXA9). Therefore, the rank product method without the sample grouping cannot identify these genes as ALL1/AF4-specific because the summary expression over all of the B-samples is so close to the T-sample levels. The box plots show the median (horizontal lines), the interquartile range (border lines), the mean (plus sign), the robust range (error bars), and the outlying observations (circles) of the expression levels of the samples in the T-cell lineage (T.NEG), as well as in the four B-cell subgroups (ALL1/AF4, BCR/ABL, E2A/PBX1, and NEG) and in their combined sample (B.SUMMARY). 
Table 4. Pathway Analysis OF THE ALL1/AF4-SPECIFIC GENES

\begin{tabular}{lccc}
\hline Gene name $^{1}$ & \multicolumn{3}{c}{ Rank } \\
MEIS1 & Rank $^{2}$ & product score & MSigDB sets \\
HOXA9 & 4 & 124.1721 & $\mathrm{a}, \mathrm{b}, \mathrm{c}, \mathrm{d}, \mathrm{e}, \mathrm{f}$ \\
PPM1H & 1 & 254.935 & $\mathrm{a}, \mathrm{b}, \mathrm{c}, \mathrm{e}, \mathrm{f}, \mathrm{g}$ \\
HOXA10 & 7 & 418.5689 & $\mathrm{f}$ \\
RHOBTB3 & 2 & 549.5536 & $\mathrm{a}, \mathrm{b}, \mathrm{c}, \mathrm{d}, \mathrm{e}$ \\
CAP2 & 18 & 568.8391 & $\mathrm{f}$ \\
DPYSL3 & - & 667.084 & $\mathrm{f}$ \\
ALOX5AP & - & 745.5577 & $\mathrm{a}$ \\
MRPL33 & - & 802.4598 & $\mathrm{~g}$ \\
HOXA5 & 17 & 814.3098 & $\mathrm{~g}$ \\
RNASE3 & - & 820.1123 & $\mathrm{a}, \mathrm{b}, \mathrm{c}, \mathrm{e}, \mathrm{g}$ \\
WT1 & - & 889.0261 & $\mathrm{~g}$ \\
CREM & - & 905.0946 & $\mathrm{a}, \mathrm{b}, \mathrm{d}$ \\
LILRA1 & - & 939.8111 & $\mathrm{f}$ \\
\hline
\end{tabular}

${ }^{1}$ The most significant genes from the ReScore procedure (FDR $<0.0001$ ).

${ }^{2}$ Rank in the original article (Chiaretti et al., 2005).

${ }^{3}$ The gene sets showing significant enrichment in GSEA $($ FDR $<0.05): a$, increased expression in NPMc + leukemias; $b$, transcription factors involved in hematopoiesis; c, genes that are upregulated in AML NPM1 mutant versus wild type; d, genes with promoter regions around transcription start site containing motif AAAYRNCTG; e, Hox genes related to hematopoiesis; $f$, genes upregulated in mouse hematopoietic stem cells and progenitors from adult bone marrow; g, genes that distinguish pediatric AML subtypes with MLL chiameric fusion genes. set $(F=0.79185)$, when compared to the conventional hierarchical clustering ( $F$-values were 0.67513 and 0.67417 with correlation and Euclidean distance, respectively). In addition to the superior overall performance, our score-based sample clustering helped us to reveal several important findings of both practical and clinical significance. For instance, four of the deep endometriosis samples were found to be mislabelled on the basis of their grouping (the asterisks in Fig. 11A) and a recheck of the surgical report confirmed that these four samples were in fact superficial peritoneal lesions.

In the control class, our analysis distinguished the peritoneal and the endometrial samples according to expectations (Fig. 11B). It was also observed that the grouping of the endometrium samples associated with the endometrial histology corresponding to the different phases of the menstrual cycle and hormonal medication, even though this information was not utilized. Of clinical interest was also the clustering of two patient peritoneum samples together with the deep endometriotic lesions, which were from patients with divergent disease condition (the arrows in Fig. 11A). It remains to be determined if these peritoneal samples included macroscopically invisible endometriosis or if the peritoneums of these patients are remarkably changed and if that will further the development of a more severe disease state. Characteristic molecular patterns behind these and many other interesting findings explaining the differences between the clinical subtypes are under further investigation.

\section{Discussion}

The key advantage of the ReScore procedure over the existing approaches was the improved capability to detect subtle alterations in the expression patterns that were shown

A

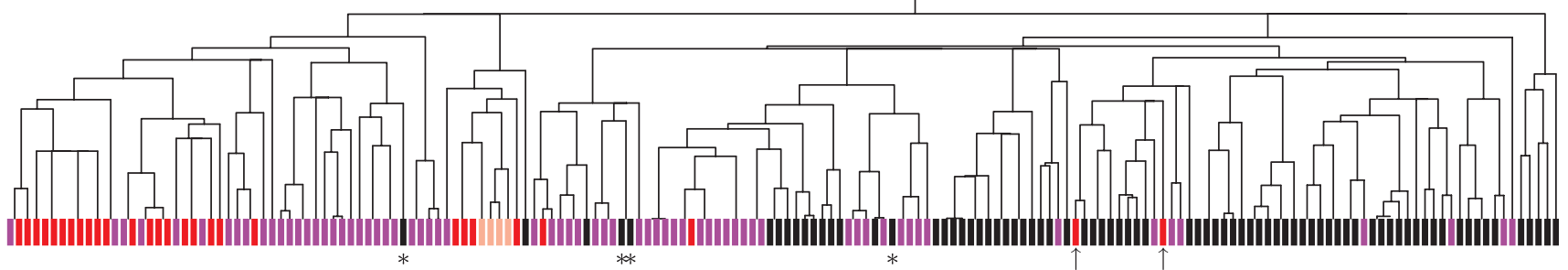

B

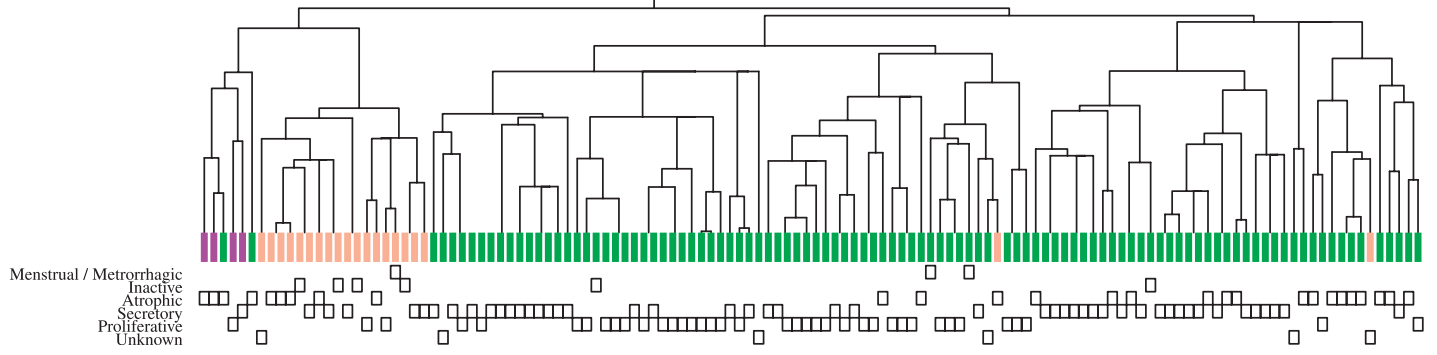

FIG. 11. Grouping of the endometriosis samples. The clustering result was split into two parts displayed on top of each other. (A) The patient samples were identified as a separate cluster, where colors indicate the origin of the sample material (red, patient peritoneum samples; magenta, peritoneal endometriotic lesions; black, deep endometriotic lesions). The asterisks below the samples indicate the four mislabelled cases discovered. The arrows indicate two interesting findings discussed in the text. (B) The grouping of the control samples was nearly perfect (pink, control peritoneum samples; green, endometrium). The boxes below the samples indicate the phase of the menstrual cycle. 
Table 5. Contribution of the ReScore Components TO THE GROUPING ACCURACY

\begin{tabular}{lccc}
\hline & $\begin{array}{c}\text { Correlation } \\
\text { coefficient }\end{array}$ & $\begin{array}{c}\text { Euclidean } \\
\text { distance }\end{array}$ & $\begin{array}{c}\text { p-norm } \\
\text { distance }\end{array}$ \\
\hline $\begin{array}{l}\text { Divisive clustering } \\
\text { Preprocessed intensity }\end{array}$ & 0.67605 & 0.66332 & 0.68647 \\
$\begin{array}{c}\text { data } \\
\text { Resampling with fold } \\
\text { changes }\end{array}$ & 0.69659 & 0.68537 & 0.72681 \\
$\begin{array}{c}\text { Resampling with rank } \\
\text { scores }\end{array}$ & 0.67108 & 0.66352 & 0.78614 \\
$\begin{array}{c}\text { Agglomerative clustering } \\
\text { Preprocessed intensity } \\
\text { data }\end{array}$ & 0.65127 & 0.63753 & 0.71913 \\
$\begin{array}{c}\text { Resampling with fold } \\
\text { changes }\end{array}$ & 0.72257 & 0.70392 & 0.74123 \\
$\begin{array}{c}\text { Resampling with rank } \\
\text { scores }\end{array}$ & 0.66412 & 0.66476 & 0.75766 \\
\hline
\end{tabular}

The relative importance of the three ReScore components (the rows) in conjunction with the three distance measures (the columns) was investigated when using either divisive clustering, or agglomerative clustering approach. The matrix entries give the grouping accuracy of the samples in the full ALL material (the F-values). The rows show the relative improvements gained by using the resampling schema and the rank transformation, whereas the columns show the effect of the $p$-norm distance. The first row is considered as reference value, and it corresponds to the standard hierarchical clustering on the preprocessed expression data (see Table 2). In the second row, the arithmetic mean was used with the fold change differences (because of the negative values), whereas the geometric mean was used with the rank scores in the third row (as in the ReScore procedure). The combination of resampling with rank scores together with the $p$-norm distance corresponds to the ReScore procedure used in the present study (third row and third column). The order $p=30$ was selected on the basis of the number of genes in the unfiltered data $(12,625$ genes). to associate with various clinically significant sample subtypes, such as genotypic abnormalities, tissue specificity, and disease modifications, hidden behind the actual phenotypic variation. The procedure is streamlined for clinical research as it can avoid making any tedious pre-processing steps, but the normalized data from the microarray experiments-warts and all — can be used as its input. For instance, the discovery of the molecularly well-defined patient subgroups could be made with much higher accuracy in the full dataset than in the filtered one (Table 2), suggesting that it is neither necessary nor even recommendable to filter down the number of genes prior to their analysis, as any type of filtering can lead to loss of important findings. Application of the ReScore to the breast cancer material (van 't Veer et al., 2002) supported these improvements illustrated here on the leukemia and endometriosis datasets, and further confirmed the benefits of the ReScoring on a wide range of clinical data and questions (see Table 1).

The relative improvements of the proposed approach can mainly be attributed to the limitations of the standard data analysis strategies. For instance, unsupervised clustering methods do not make use of the given sample labels, which may result in groupings that are unrelated to the clinical question and may even lack statistical significance. In our case studies, the unsupervised methods could not accurately detect the hidden sample subgroups, making the subsequent identification of subgroup-specific genes impossible. On the other hand, if one uses directly the expression data and the given class label information in the detection of differentially expressed genes, then many of the subsample-specific genes were missed. To be effective, supervised approaches, such as statistical inference of differentially expressed genes or feature selection in classification, would require knowledge of the hidden subgroups beforehand and hundreds of samples of each type to provide accurate results (Ein-Dor et al., 2006). Increasing sample sizes is expensive-and often even

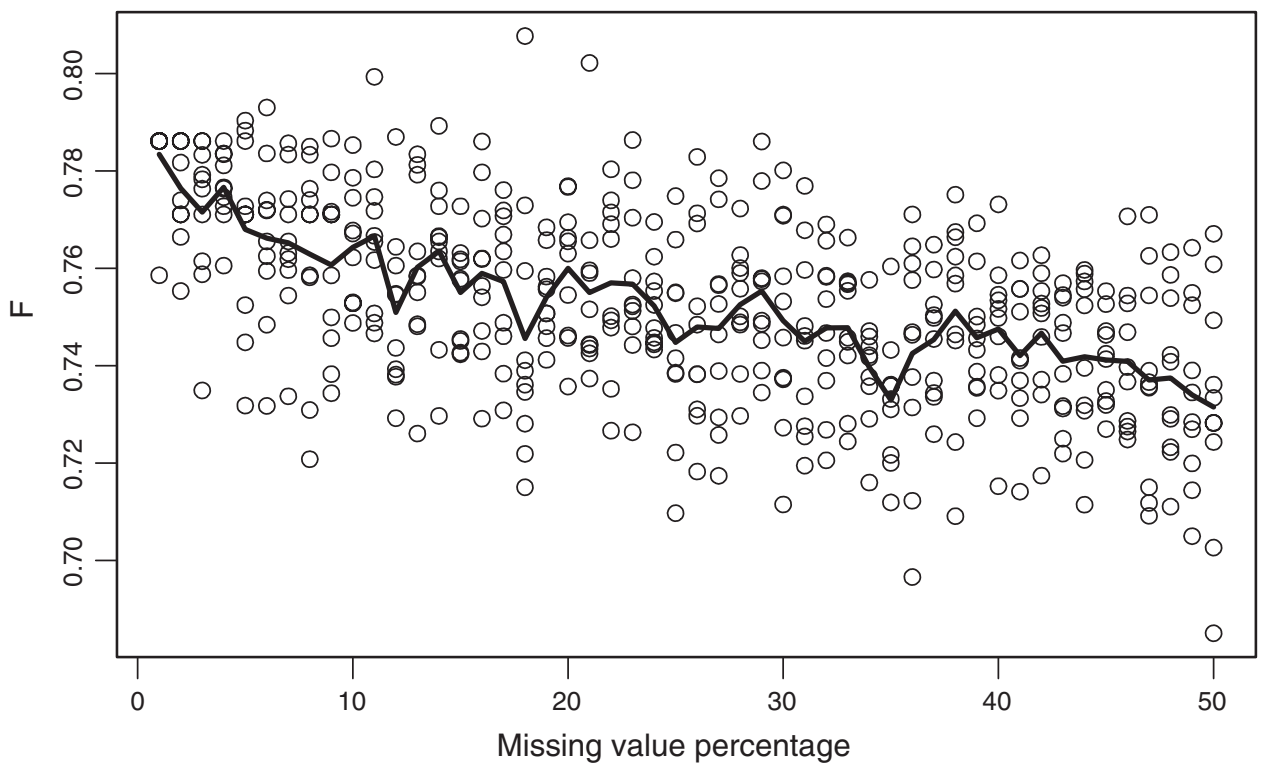

FIG. 12. The effect of missing data values on the grouping accuracy. The black line shows the mean F-level in the full ALL material ( $y$-axis) over 10 random generation of missing points (the circles) at each missing value rate ( $x$-axis). Even when $50 \%$ of the expression data values were substituted by missing values, the ReScore performed better than the best hierarchical clustering approach with no missing values $(F=0.67605$; see Table 2$)$. 


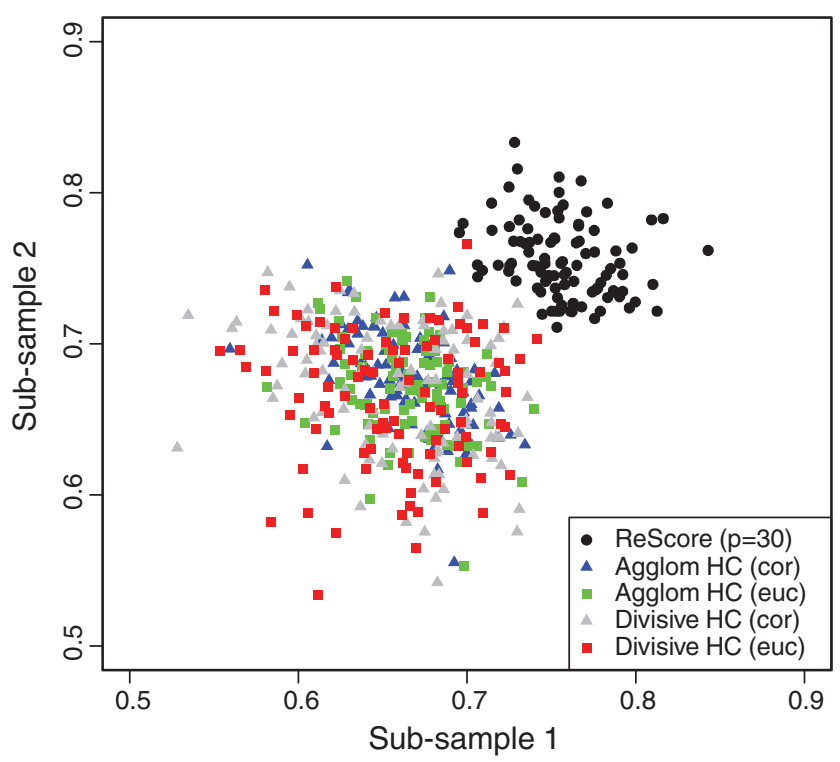

FIG. 13. The effect of sample size reduction on the grouping accuracy. The points indicate the grouping accuracy of the different methods (F-values) when the full ALL material was randomly divided into two nonoverlapping subsamples while preserving the proportion of each sample subgroup ( $x$ - and $y$-axes, respectively). Even when $50 \%$ of the original samples were omitted from the analysis, the ReScore produced superior results (average $F=0.75633$ ), whereas the accuracy of the alternative approaches was scattered around the random grouping accuracy $(F=0.66380$; see Table 2$)$. The coefficient of variation (CV) for the five approaches was $0.034820,0.045208,0.045142,0.069256$, and 0.06623 , respectively, demonstrating a slightly better agreement of ReScore across the independent subsamples.

infeasible - and can also result in additional confounders as it is impossible to control all the different factors in practice. Another limitation of the unsupervised and supervised methods when used alone is their notorious sensitivity to labeling errors and missing data (de Brevern et al., 2004; Jörnsten et al., 2005; Varshavsky et al., 2007; Wang et al., 2006).

To our knowledge, there are no other "semisupervised" methods that would share the same aims or assumptions that motivated our work. Related works include, for instance, the recently introduced surrogate variable analysis (SVA), which is another approach to deal with the heterogeneity in the expression studies (Leek and Storey, 2007). It estimates the large-scale effects of unmodeled factors from the data and incorporates them as covariates into linear models for assessing differential expression. A main difference between SVA and ReScore is the objective of the procedure, whereas the goal of SVA is to reduce the effect of the unmodeled factors to improve the detection of differential expression with respect to the known primary variable (i.e., the predefined sample groups), ReScore is designed specially for those clinical studies in which also the hidden subgroups of samples are of interest for the researcher (e.g., the distinct molecular rearrangement groups in the ALL material). Also, the methodological approaches are intrinsically different; SVA assumes a parametric model, whereas ReScore is based on a nonparametric resampling procedure, being computationally more intensive but less dependent on the properties of the dataset, such as its distributional characteristic and presence of missing values.

Even though many of its components have been successfully used separately in existing tools, the power of the ReScore procedure lies in bringing the beneficial properties of both unsupervised and supervised together under the novel resampling framework. Although the resampling schema improved markedly the grouping accuracy already with the fold-change differences, the greatest benefit was obtained after transforming these differences into the rank-based scores, especially when using the divisive clustering approach (see Table 5). However, this advance was gained only when using the $p$-norm, whereas the more commonly used distance functions, such as Pearson correlation or Euclidean distance, did not benefit from the rank-transformation. These results indicate that each of the ReScore components is required for the optimal performance of the procedure. In particular, the $p$-norm distance enabled the procedure to focus on the genes most differentially expressed in the hidden sample subgroups, whereas the resampling-based rank-scoring provided an effective way to normalize the effects of outlying samples, labeling errors and other experimental variability not directly linked to the phenotypic variability. This makes the ReScore procedure remarkably robust against missing data values (Fig. 12), and also relatively insensitive to sample size reduction (Fig. 13).

In contrast to the existing methods, the ReScore procedure can provide reliable detections in various clinical materials, without making strong assumptions about the type or amount of data under analysis. For instance, the three case studies analyzed here were conducted using different microarray platforms (Affymetrix, San Clara, CA, Illumina, San Diego, CA, and Agilent Technologies, Placerville, CA), and these datasets also represented with a wide range of intrinsic properties, such as the number of genes and samples and their distributional characteristics, which can drastically affect the performance of the traditional methods (Elo et al., 2008). Apart from the two sample classes being compared, the procedure does not require any a prior knowledge that could lead to biased results. However, the generic resampling framework could be extended to incorporate also complementary data sources, such as biological pathways (Rocke et al., 2005) or interaction networks (Chuang et al., 2007), and it could be applied also to other analysis tasks, including gene selection for diagnostic classification (Chuang et al., 2009) or to metaanalyses among multiple experiments (Higdon et al., 2008; Hong and Breitling, 2008). Although the linear selection of the order $p$ seemed to work reasonably well in these case studies, we are planning automated rules to learn the $p$-value directly from the data under analysis. Finally, the generic procedure is not limited to microarray screens only but it should prove useful also when mining clinical data from other genome-scale biotechnologies, such as those based on mass spectrometry-based quantitative proteomic assays.

\section{Acknowledgments}

The authors thank Ilana Saarikko from Genolyze Ltd. for the help with the endometriosis data, and the entire Endometconsortium for the sample material. This work was supported by the Academy of Finland (grants 120569 and 127575); 
Graduate School in Computational Biology, Bioinformatics, and Biometry (ComBi); and the Finnish Funding Agency for Technology and Innovation (Tekes grants 40343/05, 599/05, 40240/08 and 553/08).

\section{Author Disclosure Statement}

No competing financial interests exist.

\section{References}

Allison, D.B., Cui, X., Page, G.P., and Sabripour, M. (2006). Microarray data analysis: from disarray to consolidation and consensus. Nat Rev Genet 7, 55-65.

Benjamini, Y., and Hochberg, Y. (1995). Controlling the false discovery rate: a practical and powerful approach to multiple testing. J R Stat Soc B 57, 289-300.

Breitling, R., Armengaud, P., Amtmann, A., and Herzyk, P. (2004). Rank products: a simple, yet powerful, new method to detect differentially regulated genes in replicated microarray experiments. FEBS Lett 573, 83-92.

Chiaretti, S., Li, X., Gentleman, R., Vitale, A., Wang, K.S., Mandelli, F., et al. (2005). Gene expression profiles of B-lineage adult acute lymphocytic leukemia reveal genetic patterns that identify lineage derivation and distinct mechanisms of transformation. Clin Cancer Res 11, 7209-7219.

Chuang, H.-Y., Lee, E., Liu, Y.-T., Lee, D., and Ideker, T. (2007). Network-based classification of breast cancer metastasis. Mol Sys Biol 3, 140.

Chuang, L.Y., Ke, C.H., Chang, H.W., and Yang, C.H. (2009). A two-stage feature selection method for gene expression data. OMICS 13, 127-137.

De Brevern, A.G., Hazout, S., and Malpertuy, A. (2004). Influence of microarrays experiments missing values on the stability of gene groups by hierarchical clustering. BMC Bioinformatics 5, 114.

Du, P., Kibbe, W.A., and Lin, S.M. (2008). lumi: a pipeline for processing Illumina microarray. Bioinformatics 24, 1547-1548.

Efron, B, and Tibshirani, R. (2002). Empirical bayes methods and false discovery rates for microarrays. Genet Epidemiol 23, 7086.

Ein-Dor, L., Kela, I., Getz, G., Givol, D., and Domany, E. (2005). Outcome signature genes in breast cancer: is there a unique set? Bioinformatics 21, 171-178.

Ein-Dor, L., Zuk, O., and Domany, E. (2006). Thousands of samples are needed to generate a robust gene list for predicting outcome in cancer. Proc Natl Acad Sci USA 103, 59235928.

Elo, L.L., Filén, S., Lahesmaa, R., and Aittokallio, T. (2008). Reproducibility-optimized test statistic for ranking genes in microarray studies. IEEE/ACM Trans Comput Biol Bioinf 5, 423-431.

Giudice, L.C., and Kao, L.C. (2004). Endometriosis. Lancet 364 , 1789-1799.

Handl, J., Knowles, J., and Kell, D.B. (2005). Computational cluster validation in post-genomic data analysis. Bioinformatics 21, 3201-3212.

Higdon, R., Van Belle, G., and Kolker, E. (2008). A note on the false discovery rate and inconsistent comparisons between experiments. Bioinformatics 24, 1225-1228.

Holzman, T., and Kolker, E. (2004). Statistical analysis of global gene expression data: some practical considerations. Curr Opin Biotechnol 15, 52-57.

Hong, F., Breitling, R., Mcentee, C.W., Wittner, B.S., Nemhauser, J.L., and Chory, J. (2006). RankProd: a Bioconductor package for detecting differentially expressed genes in meta-analysis. Bioinformatics 22, 2825-2827.

Hong, F., and Breitling, R. (2008). A comparison of meta-analysis methods for detecting differentially expressed genes in microarray experiments. Bioinformatics 24, 374-382.

Irizarry, R.A., Hobbs, B., Collin, F., Beazer-Barclay, Y.D., Antonellis, K.J., Scherf, U., et al. (2003). Exploration, normalization, and summaries of high density oligonucleotide array probe level data. Biostatistics 4, 249-264.

Jörnsten, R., Wang, H.-Y., Welsh, W.J., and Ouyang, M. (2005). DNA microarray data imputation and significance analysis of differential expression. Bioinformatics 21, 41554161.

Kaufman, L., and Rousseeuw, P.J. (1990). Finding Groups in Data: An Introduction to Cluster Analysis (Wiley, New York). Chapter 6, pp. 253-279.

Langfelder, P., Zhang, B., and Horvath, S. (2008). Defining clusters from a hierarchical cluster tree: the Dynamic Tree Cut library for R. Bioinformatics 24, 719-720.

Leek, J.T., and Storey, J.D. (2007). Capturing heterogeneity in gene expression studies by surrogate variable analysis. PLoS Genetics 3, e161.

Michiels, S., Koscielny, S., and Hill, C. (2005). Prediction of cancer outcome with microarrays: a multiple random validation strategy. Lancet 365, 488-492.

Miklos, G.L.G., and Maleszka, R. (2004). Microarray reality checks in the context of a complex disease. Nat Biotechnol 22, 615-621.

Mootha, V.K., Lindgren, C.M., Eriksson, K.F., Subramanian, A., Sihag, S., and Lehar, J. (2003). PGC-1alpha-responsive genes involved in oxidative phosphorylation are coordinately downregulated in human diabetes. Nat Genet 34, 267-273.

Rocke, D.M., Goldberg, Z., Schweitert, C., and Santana, A. (2005). A method for detection of differential gene expression in the presence of inter- individual variability in response. Bioinformatics 21, 3990-3992.

Rotter, A., Hren, M., Baebler, S., Blejec, A., and Gruden, K. (2008). Finding differentially expressed genes in two-channel DNA microarray datasets: how to increase reliability of data preprocessing. OMICS 12, 171-182.

Sachs, L. (1996). The control of hematopoiesis and leukemia: from basic biology to the clinic. Proc Natl Acad Sci USA 93, 4742-4749.

Schmidt, A., and Aebersold, R. (2006). High-accuracy proteome maps of human body fluids. Genome Biol 11, 242.

Seo, J., Bakay, M., Chen, Y.-W., Hilmer, S., Shneiderman, B., and Hoffman, E.P. (2004). Interactively optimizing signal-to-noise ratios in expression profiling: project-specific algorithm selection and detection $p$-value weighting in Affymetrix microarrays. Bioinformatics 20, 2534-2544.

Shibata, R., Mori, T., Du, W., Chuma, M., Gotoh, M., Shimazu, M., et al. (2006). Overexpression of cyclase-associated protein 2 in multistage hepatocarcinogenesis. Clin Cancer Res 12, $5363-5368$.

Storey, J.D. (2002). A direct approach to false discovery rates. J R Stat Soc B 64, 479-498.

Subramanian, A., Tamayo, P., Mootha, V.K., Mukherjee, S., Ebert, B.L., Gillette, M.A., et al. (2005). Gene set enrichment analysis: a knowledge-based approach for interpreting genome-wide expression profiles. Proc Natl Acad Sci USA 102, 15545-15550.

Tsai, C.A., Hsueh, H.M., and Chen, J.J. (2003). Estimation of false discovery rates in multiple testing: application to gene microarray data. Biometrics 59, 1071-1081. 
Tusher, V.G, Tibshirani, R., and Chu, G. (2001). Significance analysis of microarrays applied to the ionizing radiation response. Proc Natl Acad Sci 98, 5116-5121.

Van Rijsbergen, C.J. (1979). Information Retrieval (Butterworth, London).

Van 't Veer, L.J., Dai, H., Van de Vijver, M.J., He, Y.D., Hart, A.A.M., Mao, M., et al. (2002). Gene expression profiling predicts clinical outcome of breast cancer. Nature 415, 530-536.

Varshavsky, R., Gottlieb, A., Horn, D., and Linial, M. (2007). Unsupervised feature selection under perturbations: meeting the challenges of biological data. Bioinformatics 23, 3343-3349.

Wang, S., and Cheng, Q. (2005). Microarray analysis in drug discovery and clinical applications. Methods Mol Biol 316, 49-65.

Wang, D., Lv, Y., Guo, Z., Li, X., Li, Y., Zhu, J., et al. (2006). Effects of replacing the unreliable cDNA microarray mea- surements on the disease classification based on gene expression profiles and functional modules. Bioinformatics 22, 2883-2889.

Wittner, B.S., Sgroi, D.C., Ryan, P.D., Bruinsma, T.J., Glas, A.M., Male, A., et al. (2008). Analysis of the MammaPrint breast cancer assay in a predominantly postmenopausal cohort. Clin Cancer Res 14, 2988-2993.
Address correspondence to: Dr. Tero Aittokallio Department of Mathematics University of Turku FI-20014 Turku, Finland

E-mail: teanai@utu.fi 